\title{
Die «Charta» trägt Früchte
}

Hermann Amstad ${ }^{\mathrm{a}}$, Christof Schmitz ${ }^{\mathrm{b}}$, Peter Berchtold ${ }^{\mathrm{c}}$

${ }^{a}$ Dr. med., Generalsekretär SAMW, Bern; ${ }^{b}$ Dr. rer. soc. oec., Geschäftsführer college M, Bern; ${ }^{\circ}$ PD Dr. med., Geschäftsführer college M, Bern

Im November 2014 hat die SAMW die Charta «Zusammenarbeit der Fachleute im Gesundheitswesen» veröffentlicht. Ziel der Charta war und ist es, auf das Potential der interprofessionellen Zusammenarbeit hinzuweisen und die damit zusammenhängenden Schlüsselfaktoren zu benennen. Die SAMW hat die Umsetzung der Charta mit verschiedenen Massnahmen begleitet:

- Im Sommer 2015 gab sie ein juristisches Gutachten in Auftrag, um die Reichweite der ärztlichen Verantwortung zu klären.

- Im Frühjahr 2016 führte sie in Zürich und Morges zweitägige Workshops durch zum Thema «Wie gelingt Interprofessionalität?».

- Im Herbst 2016 schrieb sie den SAMW-Award «Interprofessionalität» aus.

- Im Dezember 2016 organisierte sie das Symposium «Interprofessionalität im Gesundheitswesen: bis hierher - und wie weiter?».

- Anfang 2017 hat sie eine Auftragsstudie zur «Praxis der gelingenden interprofessionellen Zusammenarbeit» veröffentlicht.

\section{Juristisches Gutachten zur ärztlichen Verantwortung}

In der öffentlichen Debatte zur "Charta» wurde immer wieder die Frage nach der Reichweite der ärztlichen Verantwortung laut. Antworten liefert ein Rechtsgutachten, ${ }^{1}$ das die SAMW dem Institut für Gesund-

\section{Best-Practice-Beispiele haben Vorbildcharakter und können Orientierung und Unterstützung bei der Umsetzung der Interprofessionalität bieten.}

heitsrecht an der Universität Neuenburg (IDS) in Auftrag gegeben hatte. Das Gutachten klärt einerseits den Begriff «Verantwortung» und kommt andererseits zum Schluss, dass grundsätzlich jede Gesundheitsfachperson im Rahmen ihres Tätigkeitsbereichs eine Verantwortung trägt und somit auch Haftung übernimmt.

Eine Haftung für die Handlungen von Dritten ist abhängig vom Status dieser Drittperson. Das Gutachten unterscheidet drei Stufen und hält fest: je unabhängi- ger die Drittperson, desto umfassender die Haftung, die sie selber übernehmen muss; dies gilt selbstverständlich auch für die interprofessionelle Zusammenarbeit.

\section{SAMW-Award «Interprofessionalität»}

Best-Practice-Beispiele haben Vorbildcharakter und können Orientierung und Unterstützung bei der Umsetzung der Interprofessionalität bieten. Um solche Beispiele bekannt zu machen, hat die SAMW im Herbst 2016 den SAMW-Award «Interprofessionalität» ausgeschrieben für Projekte aus der Praxis, Forschung und Bildung. Auf die Ausschreibung sind 42 Bewerbungen eingegangen, 29 konnten mit dem Award ausgezeichnet werden. Die Übergabe fand Anfang Dezember 2016 im Rahmen des Symposiums «Interprofessionalität im Gesundheitswesen» statt. Prämiert wurden BestPractice-Beispiele aus allen Landesteilen und aus verschiedenen Organisationen der Versorgung, Bildung und Forschung.

Bemerkenswert sind regionale Unterschiede: Von den 11 Westschweizer Projekten kommen 7 Projekte aus dem Bildungsbereich und 4 aus der Praxis. In der Deutschschweiz ist das Verhältnis umgekehrt: 6 aus der Bildung, 9 aus der Praxis. Dies könnte bezüglich der Bildungsprojekte ein Hinweis darauf sein, dass den medizinischen Fakultäten in der Deutschschweiz ein Gegenüber fehlt, wie es in der Westschweiz mit den Fachhochschulen für Gesundheit der Fall ist.

Bei den gesamtschweizerischen Projekten sticht die «Interprofessionelle Arbeitsgruppe Elektronisches Patientendossier» hervor: Acht Berufsverbände haben sich zusammengefunden, um gemeinsam zur Realisierung von eHealth beizutragen. Dabei galt es, diverse Hürden zu überwinden, etwa unterschiedliche, berufsspezifische Begriffe für gleiche oder ähnliche Prozesse. Solche Stolpersteine zu identifizieren und aus dem Weg zu räumen ist entscheidend für eine erfolgreiche interprofessionelle Zusammenarbeit.

\section{Studie «Die Praxis gelingender Interprofessionalität»}

Die interprofessionelle Zusammenarbeit (IPZ) hat in den letzten Jahren laufend an Aufmerksamkeit gewonnen. 
Dabei wurde die Forderung nach mehr und besserer IPZ immer lauter, während die Frage offenblieb, was IPZ in der Praxis für die handelnden Fachpersonen konkret bedeutet. Dies hat die SAMW dazu veranlasst, beim college $\mathrm{M}$ in Bern eine Studie in Auftrag zu geben mit dem Ziel, dem Verständnis von IPZ in der Praxis nachzugehen und allgemeingültige Schlussfolgerungen daraus abzuleiten. An der Studie mitbeteiligt war das Institut für Soziologie an der Ludwig-MaximiliansUniversität in München. Die Ergebnisse sind in der Reihe «swiss academies reports» publiziert und auf der SAMW-Website als Download verfügbar. ${ }^{2}$

In die Studie wurden fünf verschiedene Versorgungssettings einbezogen: Grund- und Palliativversorgung, chirurgische, internistische und psychiatrische Versorgung. Insgesamt wurden 25 Interviews mit Fachpersonen unterschiedlicher Berufsgruppen in den fünf genannten Settings geführt. Im Zentrum stand die Frage, was von den Praktikern als gelingende und misslingende IPZ beschrieben wird. Daraus sollten Strategien zur Verbesserung der Zusammenarbeit der Gesundheitsfachleute abgeleitet werden.

Die Schilderungen der befragten Berufspersonen zeigen, dass die Frage, ob und wie IPZ stattfindet und ob sie von den Beteiligten als gelingend oder misslingend empfunden wird, stark davon abhängt, in welchem Kontext bzw. Setting diese Berufspersonen arbeiten. Ein Hauptbefund der Studie ist, dass die als gelungen geschilderten Beispiele von IPZ Verdichtungen bzw. intensivierte Synchronisationen von Arbeitsabläufen und Handlungen darstellen. Die Studie konnte drei unterschiedliche Modi der Zusammenarbeit verschiedener Professionen identifizieren:

- Koordinative Verdichtung meint Formen der Zusammenarbeit, in denen relativ klar definierte, meist institutionell verstetigte Handlungsmuster und antrainierte Kompetenzen ineinandergreifen. Im koordinativen Modus ist die Zusammenarbeit verschiedener Berufe oftmals durch krisenhafte Zeitknappheit oder einen relativ klar umrissenen zeitlichen Horizont gekennzeichnet (z.B. Reanimation, operativer Eingriff). Diese Form der Verdichtung lässt wesentlich auf antrainierte Kompetenzen zugreifen, die wie "programmiert» zusammengefügt werden. Programmierung und Handlungsorientierung folgen in erster Linie dem medizinischen Rational. Alle professionellen Kompetenzen sind im Selbstverständnis der ärztlichen Kompetenz komplementär beigeordnet. Beispiele dieser Verdichtungsform wurden insbesondere im chirurgischen und internistischen Versorgungssetting beobachtet. Als übergeordnetes Modell dient hier das Programm.
- Ko-kreative Verdichtung meint Formen der Zusammenarbeit, die auf ein Nach- und Nebeneinander verschiedener professioneller und individueller Kompetenzen setzt, die in relativ weiten Zeithorizonten diskursiv aufeinander abgestimmt werden (z.B. in der palliativen Betreuung). Die verschiedenen Professionen können nicht anders als gemeinsam und mit dem Patienten Lösungen suchen und entscheiden. Keine Profession kann per se Dominanz beanspruchen. Asymmetrien und Entscheidungskompetenzen sind von deren alltagspraktischer Aushandlung und Bewährung abhängig. Eine organisationstheoretische Entsprechung findet die ko-kreative Zusammenarbeit im Modell des Netzes mit den entsprechend verteilten Formen von Deutungshoheit.

Die Frage, ob IPZ von den Beteiligten als gelingend oder misslingend empfunden wird, hängt stark davon ab, in welchem Kontext bzw. Setting diese Berufsgruppen arbeiten.

- Zwischen den beiden Polen des Koordinativen und Ko-Kreativen liegt eine breite Zone dessen, was in der Studie als projekthafte Verdichtungen bezeichnet wird. IPZ findet sich im Gelingensfall als temporär fungierende oder anspruchsvoll organisierte Projekte verdichteter Zusammenarbeit. Beispiele können von Runden Tischen in der Grundversorgung über interprofessionelle Tumorboards oder Fallführungen bis hin zu anspruchsvollen Organisierungsformen im Sinne einer achtsamen, wechselseitigen Bezugnahme (heedful interrelating) in der Psychiatrie reichen. Organisationstheoretisch liegt hier zur Einordnung das Modell des Knotens nahe.

Die Schilderungen der Befragten bringen weiter zum Ausdruck, inwiefern (a) die Medizin mit ihren professionellen Selbstverständnissen und Kompetenzen die Deutungshoheit innehat, (b) die Individualität des Patienten, der Patientin einfliesst und (c) die spezifische Fachkompetenz und Individualität der Gesundheitsfachleute ins Spiel kommen oder gar leitend werden. Exemplarisch und polar stehen sich hier Reanimation und Palliation gegenüber: Im einen Fall folgt die Zusammenarbeit ganz medizinischen Handlungslogiken, im anderen Fall strukturiert sich die Zusammenarbeit über mehrdimensionale Aushandlungsprozesse zwischen der Patientenperspektive und den verschiedenen professionellen Perspektiven. Offensichtlich kann das Verhältnis dieser beiden Dimensionen variieren. Abbildung 1 zeigt, wie die 


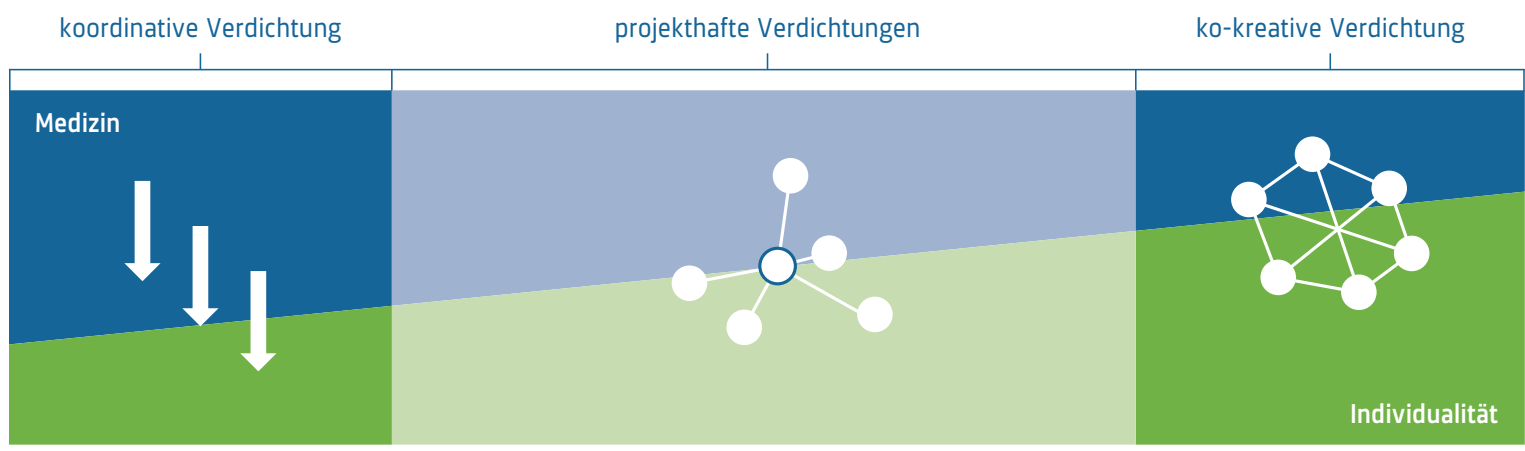

Abbildung 1: Interprofessionelle Zusammenarbeit: Verdichtungsformen, Verhältnis Medizin-Individualität und Organisationsmodi.

drei Verdichtungsformen und das Verhältnis Medizin-Individualität zueinander in Beziehung stehen.

Die Studie kommt zum Schluss, dass Verdichtungen interprofessioneller Zusammenarbeit - den Wahrnehmungen und Erzählungen der befragten Fachpersonen zufolge - zunächst vor allem um aktualisierte Krisen von Patienten entstehen. Dabei unterscheiden sich

\section{Unbestritten sind eine konstruktive Zusam- menarbeitskultur und Augenhöhe zwischen den Berufsgruppen eine wichtige Voraus- setzung für gelingende IPZ.}

die Arten von Krisen und die durch sie provozierten Verdichtungsformen interprofessioneller Zusammenarbeit stark. Akut-somatische Krisen etwa koordinieren die Expertise der verschiedenen Fachpersonen entlang einer medizinischen Logik; Lebensendkrisen eines Palliativpatienten provozieren individualisierte Behandlungspfade, ohne sich einem einzelnen Rational zu fügen.

Während sich diese beiden Verdichtungsformen um konkrete Fälle bzw. Patienten bilden, ist die dritte, die projekthafte Verdichtung, situationsunabhängiger. Sie handelt von Prozessen punktueller oder auch übergreifender Organisierung, die dazu dienen, Patienten, die mehrere Disziplinen und Professionen erfordern, besser begegnen zu können. Die projekthafte Verdichtungsform kommt dann ins Spiel, wenn man sich nicht damit zufriedengeben will, dass ein schwach koordiniertes, komplementäres Nebeneinander von Kompetenzen - der «Normalfall» der Gesundheitsversor- gung - bereits hinreicht. Projekthafte Verdichtungen variieren breit und finden sich in vielfältigen Settings.

\section{Fazit und Ausblick}

Unbestritten - und von allen Befragten nachdrücklich betont - sind eine konstruktive Zusammenarbeitskultur und Augenhöhe zwischen den Berufsgruppen eine wichtige Voraussetzung für gelingende IPZ. Gleichzeitig machen die oben geschilderten Studienergebnisse deutlich, dass ein kultureller Wandel einen nötigen, aber keinen hinreichenden Ankerpunkt bildet, um interprofessionelle Zusammenarbeit nachhaltig zu fördern. Die Anerkennung von und der Abgleich mit den spezifischen Anforderungen der jeweiligen organisatorisch und fachlich definierten Settings ist ebenso zentral.

Zweitens, nimmt man die Erfahrungen der befragten Berufspersonen ernst, ist IPZ nicht mit der (Neu-)Verteilung von Aufgaben zwischen den Berufsgruppen oder mit Delegation bzw. Substitution gleichzusetzen. Die Verschiebung von Aufgaben zwischen Berufsgruppen - so angebracht sie aus verschiedenen Gründen sein kann - tangiert nicht automatisch das beschriebene Moment der Verdichtung von Arbeitsabläufen und Handlungen. Anzumerken bleibt allerdings, dass die Thematisierung und Implementierung solcher Neuverteilungen ausgezeichnete Anlässe für IPZ-relevante Reflexionen geben können.

\section{Bildnachweis}

Abbildung aus der Studie «Die Praxis gelingender Interprofessionalität», siehe Fussnote 2 . 